\title{
Design and Development of Secured Communication Between Smart Meter and Neighborhood Gateway
}

\author{
Kodamala Venkatesulu' ${ }^{1}$, Dr. G. Mamatha ${ }^{2}$
}

${ }^{1}$ Department of ECE, JNTUA College of Engineering, Ananthapuramu, Andhra Pradesh, India

${ }^{2}$ Assistant Professor, Department of ECE, JNTUA College of Engineering, Ananthapuramu, Andhra

Pradesh, India

\begin{abstract}
A smart meter is an advanced meter which measures power consumption in much more accurately than a conventional meter and communicates the collected information back to the usage for load limit and tariff purposes. The objectives are privacy that nobody can obtain power usage of other person's information if the protocol is accurately executed. Real time authentication that transmitted message can be real timely authorized by the receiver which is essential to resist against the denial of service (DoS) attack, Replay attack resistance that receiver can validate whether the received messages are the replay of previously authorized persons. The main objective of this new technology is the bidirectional flow of information. The smart meters send the power consumption reports to the power operator and also control instructions are sent from electricity board in order to be executed by the smart meters. In between, there consists of some gateways which are responsible for data accumulation. The main objective of the system is the communication of smart meter and neighborhood gateway. The presented communication scheme must consider the necessity for consumption reports transmission in short time intervals, and also it must consists of both security and the limited resources of smart meters. This implemented system demonstrates substantial reduction in storage space and data modifications are avoided.
\end{abstract}

Keywords : Smart Meter, Neighbourhood gateway, DoS, protocol, Reply Attack, Authentication.

\section{INTRODUCTION}

India is the one of the biggest countries in the planet has one billion families with electric meters. These electric meters are working manual and meter readings are collecting manually. An individual takes the meter reading using measurement devices and then it is to be submitted to the power operator office. This process consists of errors and reading problems. Electricity has turned out essential in life. But the issues of electricity have implied that people consume large amounts of energy ignorantly and recklessly.
Smart Grid got consideration from both scholarly world and industry because of its many amazing features, The features are self-healing, two-way communications, remote check, distributed management and control, and more reliable, sustainable, and economical electricity is the goal of the implementation. In smart grid, the communications are bidirectional and in coming days, the customers can infuse their energy back to the power grid. Smart meters are usage reporting devices; they will send the electricity consumption data to the power operator through some intermediate gateways 
that act as data collectors. In return, some instructions are sent from the power operator to the smart meters through the neighborhood gateways. The frequent gathering of utilization data may divert the privacy of consumers. If an attacker collects these reports, he/she will be able to retrieve the secret and private data of consumers, such as deciding the inhome presence, in-home exercises, and individual habits. For example, if the utilization data, for an instance of time, are low and constant, it will be known that nobody is at home. The neglect of this imperative subject will straight forwardly influences the acceptability of and will slow down the up gradation of the present technology. The real-time power consumption data collection from consumers not only makes real-time monitoring and control much more accurately, but also encourages the consumers to decreasing their power utilization cost by decreasing the peak time utilization. It will help the power operator to better predict the near future demands of consumers and will lead to the efficient utilization of the energy resources and power stations.

\section{EXISTING SYSTEM}

The existing system of energy metering utilizes electromechanical and digital energy meters have poor exactness and absence of configurability and also needs more time and work. Presently number of advanced electronic interface meters has been introduced. These advanced meters can store, process and communicate utilizing digital data formats. But the data will be sent for each 5 to 15 minutes. So the energy meter needs to store it. It will become a burden for energy meter. And also there is a probability of meter's memory modification. So, it is a problem for electricity board. The processed information was transmitted utilizing wireless technologies such as Zig Bee network protocol. But $\mathrm{Zig}$ Bee is a short range wireless protocol. So, a better wireless network is to be proposed. The post paid energy meters allow the consumer to utilize more amount of electricity than required. It will become a problem for a consumer for paying power bill.

\section{IMPLEMENTED SYSTEM}

The implemented system mainly deals with smart energy meter, which uses the features of embedded system combination of hardware and software in order to design our functions. Using Wi-Fi modem the customer can control his utilized consumption reading and can upgrade the threshold value through the webpage. This system continuously collects the power consumption data and the live meter reading can be displayed on the webpage to the customer on request. The transmission of collected information is in real time basis. It won't require burden for storing information in meters. This system can be utilized to switch off the power supply of the house when needed.

\section{Black diagram}

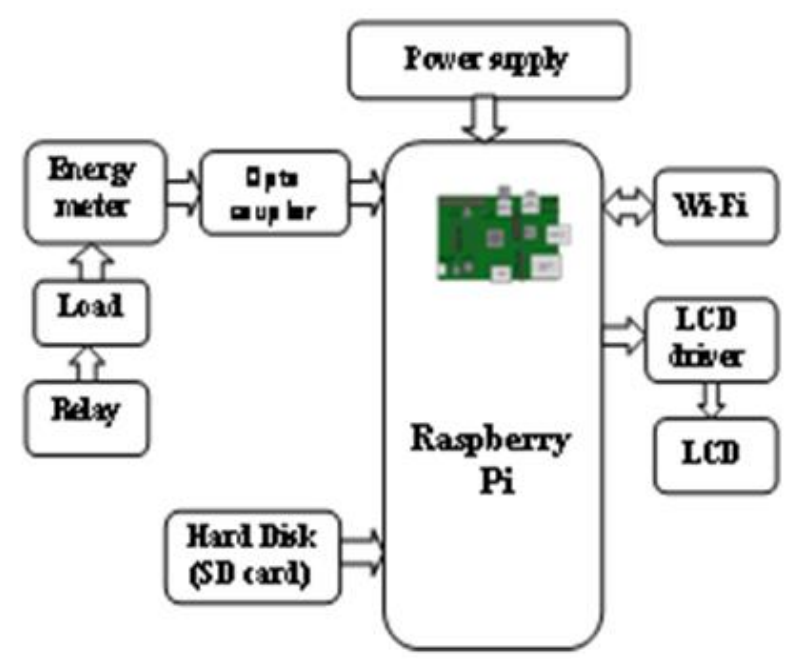

\subsection{WORKING OF IMPLEMENTED SYSTEM}

- When the different appliances of the household uses energy, the energy meter collects the reading continuously and this utilized load can be shown on energy meter.

- The LED on energy meter consistently blinks according to the energy meter. Based on the 
number blinks, the units are counted. Usually, 3200 blinks are taken as one unit.

- In this system, we developed a system in which Raspberry $\mathrm{Pi}$ acts as a main controller, which continuously monitor energy meter.

- According to the blinking of LED on energy meter, the Raspberry Pi will measure the unit power consumption.

- Threshold value can be upgraded on webpage through the $\mathrm{Wi}-\mathrm{Fi}$, according to the consumer's requirement. When the consumer's power consumption reading crosses the threshold value, it will send a notification value to the consumer.

- The consumer needs to visit the webpage to upgrade the threshold value. By upgrading the threshold value, the energy meter will automatically get $\mathrm{ON}$.

- The power consumption data and the control instructions like load limit and the threshold values will be encrypted before transmission.

- The received encrypted data will be decrypted and showed on the display.

\subsection{WORKING FLOW}

\section{Flow chart}

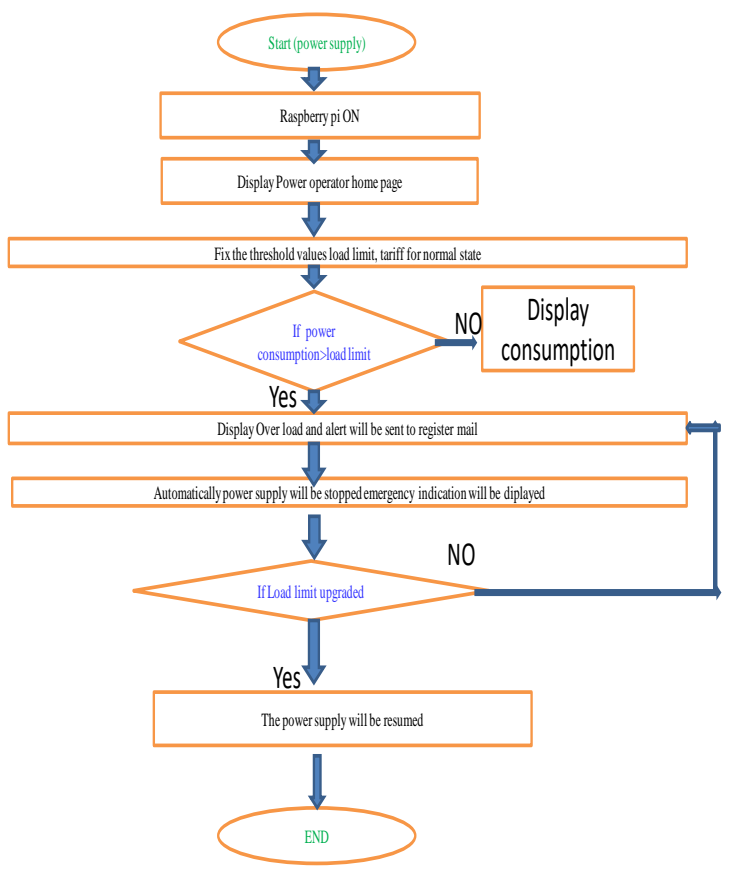

\section{HARDWARE WORKING DESCRIPTION}

The brief introduction of working of different modules used in this project is discussed below:

\section{1 ENERGY METER -AD7751 IC}

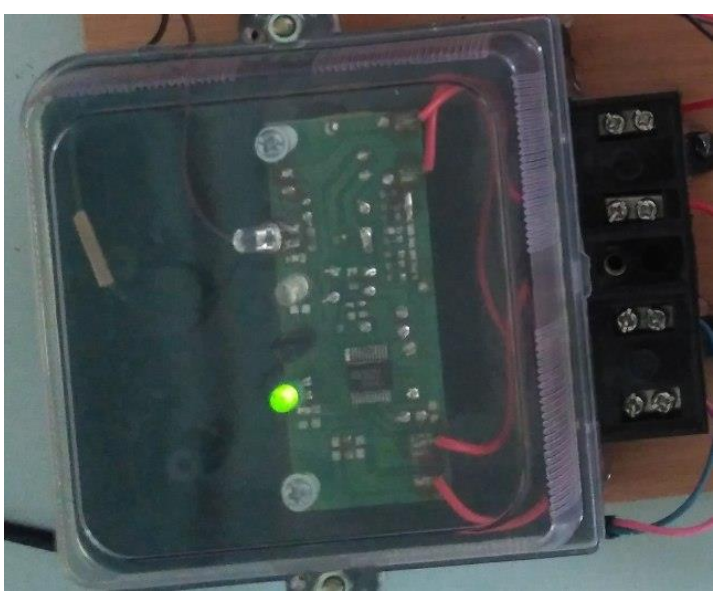

Fig 1. AD7751 IC-Energy meter

The AD7751 is a high accuracy energy measuring IC. The Energy meter will collect the power consumption report consistently. The LED will blink in accordance with the energy meter reading. The LED emits the $0.7 \mathrm{~V}$ as a pulse. These pulses are fed to the Raspberry Pi through Opto-coupler. The LED will blink slowly whenever power consumption at a particular time is low. If the power consumption is high then the LED will blink fast. The energy meter will not store any measured value. It will be sent as a pulse in real time manner.

\subsection{RASPBERRY PI (ARM-11) PROCESSOR}

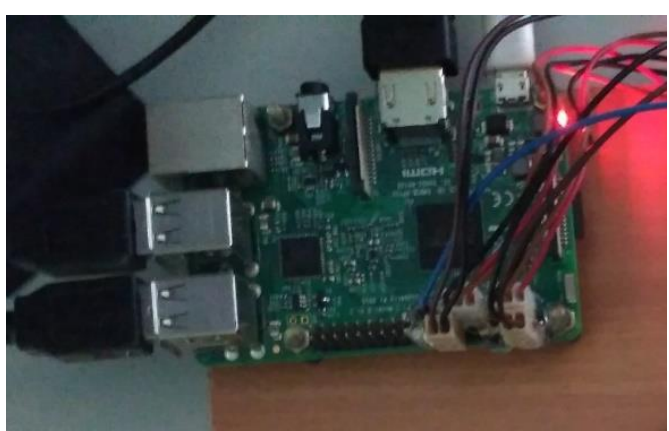

Fig 2. Raspberry pi3 used in system 
Raspberry pi is the heart of our system. Entire functioning of system depends on this board. Raspberry pi monitors the $5 \mathrm{v}$ supply sent by Optocoupler and continuously counts the pulses and then calculates the electricity used and also the peak time usage. This reading will be stored on webpage in real time. The consumer can see their stored data at any time through webpage and check their electricity usage data. The complete control and monitoring of energy meter and Gateway will be done by Raspberry Pi.

\subsection{OPTO-COUPLER}

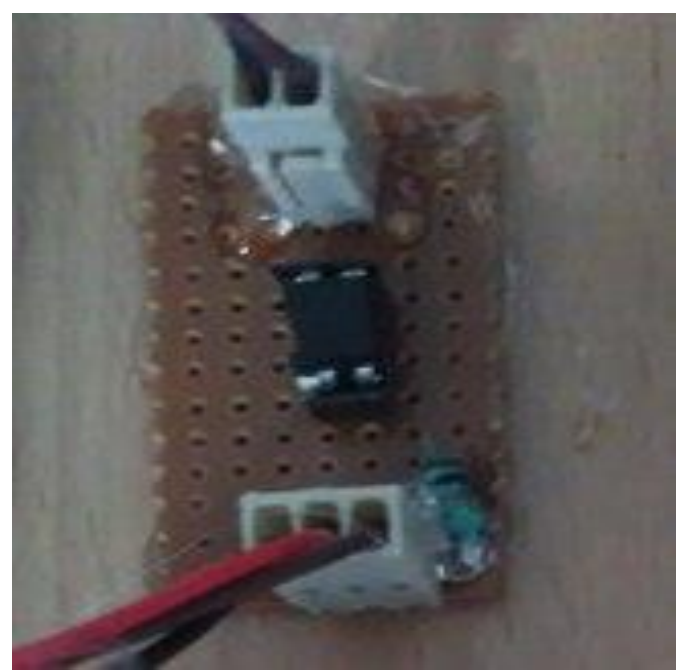

Fig 3. Opto-coupler

One of the LEDs on energy meter will continuously blink; it indicates the count of power. Whenever the LED blinks it generates only $0.7 \mathrm{v}$ which is not sufficient for Raspberry pi to collect, so to avoid this error we are placing Opto-coupler in between energy meter and Raspberry Pi. When the LED blinks the diode will conduct, the generated $0.7 \mathrm{v}$ will make transistor to work in active region, and then the output will be $5 \mathrm{v}$, which is given as power supply for transistor. As LED blinks, the 5v supply will be given to Raspberry pi and then it will count those pulses. We are using Opto-coupler to increase voltage from $0.7 \mathrm{v}$ to $5 \mathrm{v}$, and then only the Raspberry Pi can monitor the pulses.

\subsection{RELAY}

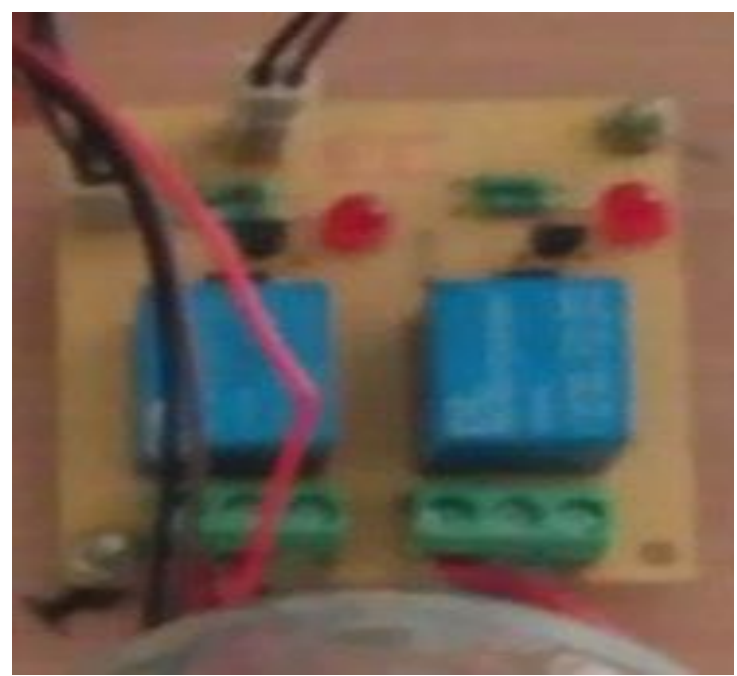

Fig 4. Relay module

A relay is an electrically working switch. A relay uses an electromagnet to work as a switch. Whenever consumption data is within limit, it is connected to the normally closed (NC) of the relay and whenever threshold value crosses the limit then the switch goes to the normally Open (NO) contact. The relay operation is useful for denoting the load and the emergency alert notifications. The primary relay is to denote the emergency alert, and the secondary relay for connecting and disconnecting the household supply.

\subsection{WEB PAGE (HTML)}

We designed webpage for operating Raspberry pi and Energy Meter with the help of HTML. The threshold values like load limit and tariff will be upgraded form the webpage. If the consumer's power consumption reading crosses the load limit, the power operator will upgrade the load limit to a new value on request. The stored data like consumption report, tariff and load limit can be seen on the webpage.

\section{SECURITY ALGORITHM USED}

To achieve the security for the communication, we used AES 128 bit Security algorithm. In this system, 
the security algorithm enables the authentication whether an authorized consumer getting information from the power office or not. The power consumption report and the control instructions will be encrypted to avoid divulge of information. The encrypted data will be communicated between smart meter and the neighborhood gateway. In return the control instructions will be sent back to the smart meter from the gate way.
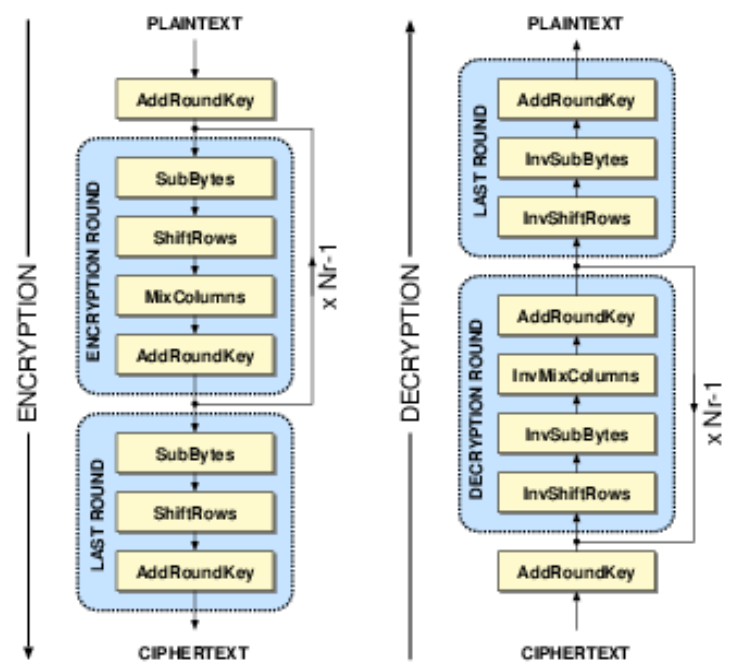

Fig 5. AES algorithm encryption and decryption

\section{EQUATIONS AND MATHEMATICAL CALCULATION:}

Usually different meters have different readings.

1500 blinks $=1$ unit or

3200 blinks = 1 unit as per designer.

Let us assume,

3200 blinks = 1 unit of reading.

Let, $\mathrm{X}=$ No. of blinks of LED

$\mathrm{Y}=$ No. of units of power consumption.

$\mathrm{Z}=$ cost of electricity.

Normally,

Number of units $(\mathrm{Y})=(\mathrm{X} / 3200)$

Let us assume,

1 unit cost $=3.0$ Rs

$\mathrm{Z}=\mathrm{Y}^{*} 3.0 \mathrm{Rs}$.
For Threshold value,

Assumed threshold set value will be $=0.006$ units for practical.

If units reach threshold value the overload condition will occur and then meter will automatically get OFF.

Then consumer has to visit webpage again to increase threshold value.

\section{VII.RESULTS}

The development of "Design and development of secured communication between smart meter and neighbourhood gateway" aims to develop a security system for energy meters. The system is able to send power consumption reports to the gateway and instructions are sent back to the smart meters in an encrypted form. The authentication of sender and receiver is performed using the security algorithm. The implemented system sends alerts to the consumers when load limit crosses. And then if the load limit will be upgraded, then power supply will be restarted. The below images show the proposed system and its output.

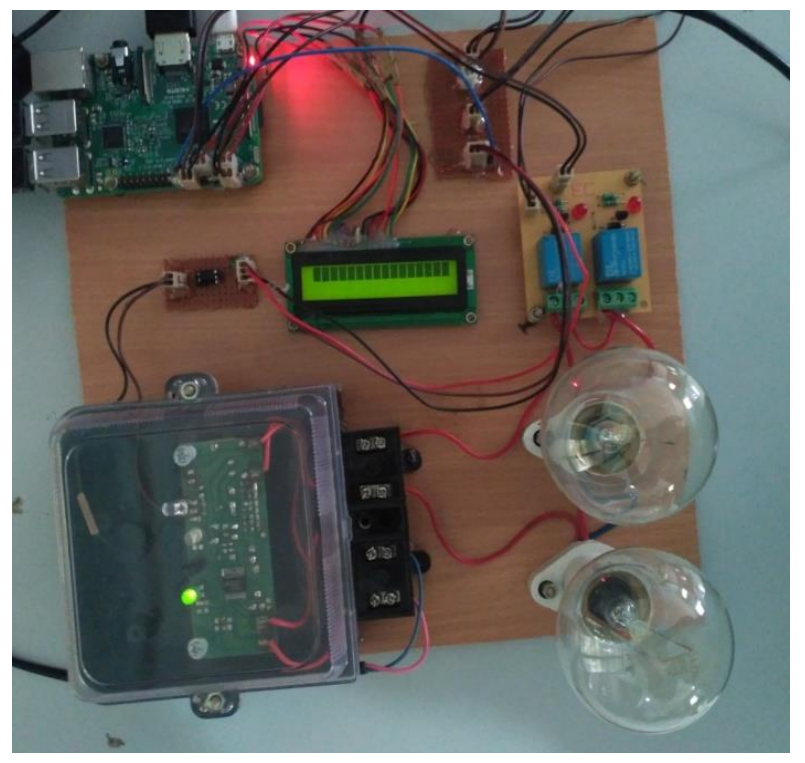

Fig 6. Secured communication between Smart meter and Neighborhood gateway 


\subsection{WEB PAGE -REAL TIME DATA}

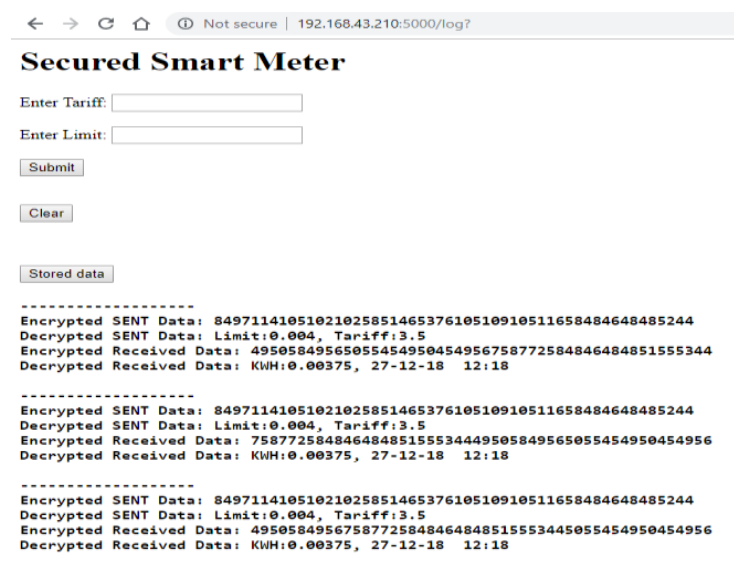

6.2 CONSUMER TRACKING DISPLAY
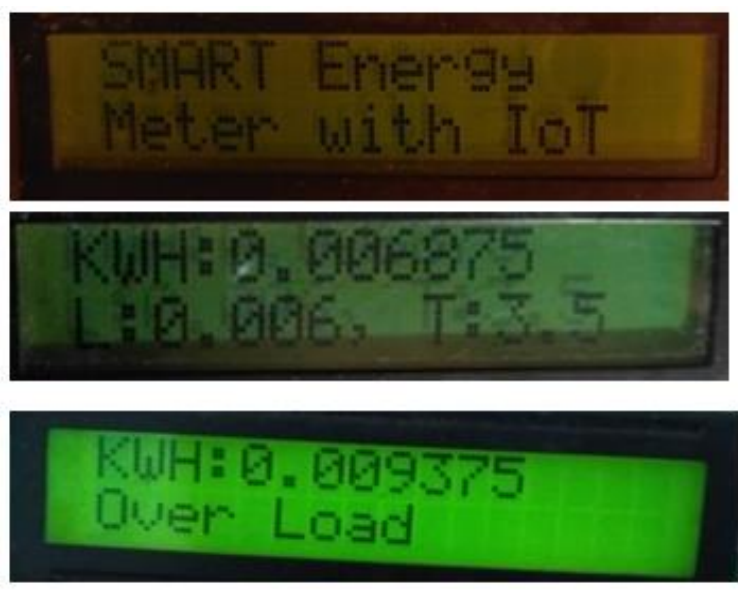

6.3 MAIL ALERT

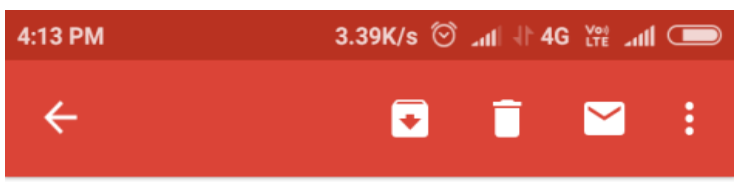

Alert. LOAD Limit Crossed: 05-12-18 16:12 Inbox

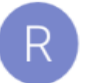

raspberry1786@yahoo.in \& :

to me

4:12 PM View details

You have crossed Load Limit. Please contact AE for upgrade.

\section{CONCLUSION}

The secured communication between smart energy meter and gateway was Designed which uses bidirectional flow information. Real time monitoring and decision making will be possible as usage reports transmission is in real time. The model is designed to work with a two lamp load. The consumer data can be communicated with high security. The modification of meter readings can be avoided with the implemented system. The errors, time delay that are faced because of manual metering can be avoided. The implemented system presents a combined attribute of all the hardware components which has been used and developed in it with Arm-11 Raspberry pi processor. Using highly advanced IC's like 1.2GHz Quad-Core ARM Cortex-A53 (64Bit) processor, AD7751 IC for energy meter, Opto coupler, Linux operating system technology with the help of growing technology. Thus, the project has been successfully designed and tested.

\section{ACKNOWLEDGEMENTS}

The first person I would like to thank is my guide Dr.G.Mamatha, assistant professor of Electronics and Communication Engineering Department, JNTUA College of Engineering, Anantapuramu. Her wide knowledge and logical way of thinking have made a deep impression on me. Her understanding, encouragement and personal guidance have provided the basis for this thesis. She is a source of inspiration for innovative ideas and her motivation.

\section{REFERENCES}

[1]. Dariush Abbasinezhad-Mood and Morteza Nikooghadam, "An Ultra-Lightweight and Secure Scheme for Communications of Smart Meters and Neighborhood Gateways by Utilization of an ARM Cortex-M Microcontroller", $\quad$ DOI 10.1109/TSG.2017.2705763, IEEE Transactions on Smart Grid, 1949-3053 (c) 2016 IEEE

[2]. S. Uludag, K.-S. Lui, W. Ren, and K. Nahrstedt, "Secure and scalable data collection with time 
Minimization in the smart grid," IEEE

Transactionson Smart Grid, vol. 7, no. 1, pp. 4354, Jan. 2016.

[3]. Y. Liu, C. Cheng, T. Gu, T. Jiang, and X. Li, "A lightweight authenticated communication scheme for smart grid," IEEE Sensors Journal, vol. 16, no. 3, pp. 836-842, Feb. 2016.

[4]. K. Mets, J. A. Ojea, and C. Develder, "Combining power and communication network simulation for cost-effective smart grid analysis," IEEE Communications Surveys \& Tutorials, pp. 1771-1796, 2014.

[5]. H. Wang, B. Qin, Q. Wu, L. Xu, and J. Domingo-Ferrer, "TPP: Traceable PrivacyPreserving communication and precise reward for vehicle-to-grid networks in smart grids," IEEE Transactions on Information Forensics and Security, vol. 10, no. 11, pp. 2340-2351, Nov. 2015.

\section{Cite this article as :}

Kodamala Venkatesulu, Dr. G. Mamatha, "Design and Development of Secured Communication Between Smart Meter and Neighborhood Gateway", International Journal of Scientific Research in Science and Technology (IJSRST), Online ISSN : 2395-602X, Print ISSN : 2395-6011, Volume 6 Issue 1, pp. 378-384, January-February 2019. Available at doi : https://doi.org/10.32628/IJSRST196157 